\title{
Exploiting Symmetry and Transactions for Partial Order Reduction of Rule Based Specifications ${ }^{\star}$
}

\author{
Ritwik Bhattacharya ${ }^{1}$, Steven M. German ${ }^{2}$, and Ganesh Gopalakrishnan ${ }^{1}$ \\ 1 School of Computing, University of Utah \\ \{ritwik, ganesh\}@cs.utah.edu \\ 2 IBM T.J. Watson Research Center \\ german@watson.ibm.com
}

\begin{abstract}
Rule based specifications are popular for specifying protocols, e.g., cache coherence protocols specified in TLA+ [12], Murphi [7], or the BlueSpec language [1]. Specifications in these notations are a collection of unordered rules of the form guard(state) $\rightarrow$ atomic_updates. There is no notion of a sequential process with local scopes or channels, and each rule tends to update multiple fields of the global state. It is believed that partial order (PO) reduction is difficult to achieve in such a setting. In our preliminary work $[2]^{1}$ we reported a suitable algorithm for this purpose. In this paper, we expand on this algorithm and show that this algorithm can exploit the transactional nature of many protocols in this area, during ample-set computation. Second, we show that, in the presence of symmetry, the SAT-based computation of the independence relation between rules can be computed once and for all in a manner that is accurate for all parameterized instances of the protocol; Third, we show that sharpening the SAT-based independence computation through local invariants can aid PO reduction. Here, we propose a way by which users may guess these invariants: we can check these invariants and the property of interest in one combined phase under PO reduction (we prove that there is no circularity in this process). Our results indicate that with the above measures, rule based systems can have efficient and effective PO reduction algorithms.
\end{abstract}

\section{Introduction}

Rule-style specification of protocols are widely employed. They are often written in languages such as Murphi, TLA+, or BlueSpec, and are often used for modeling cache coherence, file systems, message networks, and solutions to similar locking/concurrency problems. Protocols specified in these notations are a collection of unordered rules of the form guard(state) $\rightarrow$ atomicUpdates. The

\footnotetext{
^ This work was supported in part by NSF Award CCR-0219805, and SRC Contract 2005-TJ-1318

${ }^{1}$ Short paper of four pages.
} 
state of systems modeled in such notations can be a global aggregate datatype such as a record of arrays of simpler types or other records. There is no notion of a sequential process with local scopes or FIFO channels 2 , and each rule tends to update multiple fields of the global state. Such specifications are natural to write for domains such as cache coherency, where designers try to have a declarative approach to modeling using an unordered collection of rules (they would often think as follows: "under condition X, emit the set of actions Y.") Hardware systems often have massive amounts of concurrency. It is natural to view this concurrency in terms of collections of rules, where many rules may update the same part of the global state space. In contrast, it can be difficult and unnatural to partition a model of a hightly concurrent hardware system into a small number of interacting processes having disjoint local state. Such unordered collection of rules are often automatically compiled into the underlying cache coherency engine [1; from this perspective, the unordered and declarative nature of the rules leads to concurrent hardware that can be modularly understood.

SAT based independence computation, exploiting local invariants: Given all this, however, it is clear that one of the main weapons to combat state explosion of these protocol models during enumerative model checking - namely partial order reduction (PO reduction or POR) - becomes difficult to realize. For example, consider the two guard::action pairs below:

1. $((i>=0):: a[i+1]:=$ True $)$

2. $((i<=0):: a[i+2]:=$ False $)$

A syntax-based PO reduction approach (such as used in SPIN) would classify rules 1 and 2, which both access the same array variable, as dependent. Our symbolic simulation based approach would, on the other hand, determine that the rules are independent, since in all states where both rules are enabled, the rules access different indices of the array a. In particular, our independence computation based on SAT will, as most used definitions of independence require (e.g., 6. Chapter 10]), (i) start the system from a general symbolic state $s$ (all states are broken into the individual bits), (ii) pick a pair of rules $r_{1}$ and $r_{2}$, (iii) determine whether, whenever $r_{1}$ and $r_{2}$ are enabled in $s$, firing one rule leaves the other enabled, and (iv) determine whether $r_{1}\left(r_{2}(s)\right)=r_{2}\left(r_{1}(s)\right)$. All this is performed using SAT. For example, the last step is realized by seeing whether $r_{1}\left(r_{2}(s)\right) \neq r_{2}\left(r_{1}(s)\right)$ can be satisfied.

Obviously, doing this analysis starting from a general starting state can result in pessimal independence information. Later we show how local invariants can help sharpen the analysis to states that contain reachable states but exclude certain unreachable states. One of our main results is that the kinds of invariants that tend to give sharp results regarding invariants are not those that we are

\footnotetext{
${ }^{2}$ While FIFO channels are convenient for modeling, and help obtain the benefits of PO reduction, (i) rule based languages we know about do not support channels, and (ii) designers often want something other than any one of the standard varieties of channels such as FIFO, sorted, lossy, etc.
} 
proving at the top level (e.g., cache coherence), but those that (i) provide some information about which rules might follow which other rules, and (ii) those that tend to capture relationships between variables that the protocol writer tends to use. More specifically, we observe from the available list of benchmark protocols written by others that one often employs more variables than necessary in a protocol for modeling convenience. Our observation is that often it is necessary to pin down relationships that might exist between these variables. In any case, once a local invariant $g_{1}$ is obtained, a rule such as $g \rightarrow$ action is strengthened into $g \wedge g_{1} \rightarrow$ action. We show that such strengthened rules yield a better (larger) independence relation.

Clearly, we do not wish that the solving the problem of POR lead to another problem, namely that of invariant discovery! Here, we have found a less painful approach. We show a method by which designers can (i) guess these invariants (even if they are incorrect, we will be safe, as we show below), (ii) perform the independence check with respect to modified rules of the form $g \wedge g_{-} 1 \rightarrow$ action, but (iv) while performing model checking using our POR algorithm (that of course enjoys the benefit of the larger independence relation), be checking not merely original_property but actually original_property $\wedge\left(g \Rightarrow g_{1}\right)$. If there are more guards than one strengthened in this way, for each such $g$ and its strengthening $g_{i}$, we would have the conjunct $g \Rightarrow g_{i}$ in the top-level property being verified. This way, if any of the $g_{i}$ excludes a state that $g$ includes, it will be detected while verifying the modified property that includes $g \Rightarrow g_{i}$.

Symmetry and Carry-over of Independence Computation: We observe that many of the protocol descriptions in languages such as Murphi employ scalarsets [11. Furthermore, the variables that are of type scalar set participate in ruleset constructs inside protocols. For example, if a cache coherence protocol has $N$ nodes, it is quite likely that there is a ruleset collectively modeling each node's behavior. In the Murphi model checker, rulesets defined over scalar set parameters are handled as follows: (i) the user picks a number (e.g. 4) for the size parameter of the rule set, (ii) the model-checker creates four copies of the rules, and (iii) while model checking, these four rules are non-deterministically fired in all possible ways (this is necessary, as each rule instance may be, for example, modeling the behavior of a different caching node), (iv) after each state is generated, Murphi's symmetry algorithm performs state canonicalization [11, thus generating representative states out of each.

Our approach to exploit the scalarset symmetry is as follows: (i) we demonstrate that under certain conditions, we can, for the purposes of SAT-based independence computation, analyze an instance of size $N$ but model-check an instance $M>N$. This way, the number of rules analyzed as well as the data structures involved in the analysis (e.g., if the data structure sizes were determined by $N$ ) would be much smaller. (ii) later, the user may model-check an instance $M$ that is much bigger than $N$, because currently parameterized proofs are hard to obtain and the designer takes the approach of flushing out bugs in as high an instance as they can. However, in model checking the $M$-sized instance, 
the user can employ the same independence matrix as calculated on the basis of an $N$-sized instance.

Ample Set Computation Exploiting Transactions: In ample set based POR algorithms (e.g., [6, Chapter 10]), computing the independence relation is only part of the story; ample set formation is a run-time activity where independence comes into play. Ample sets are minimal (subject to certain sufficient conditions) subsets of the set of enabled transitions at a state, and act as a locally optimal heuristic to maximize global state space reduction. Naturally, smaller ample sets are preferred over larger ones. In our approach, ample sets are computed by picking a seed transition and performing a least-fixed-point computation based on the dependence relation. We then check to ensure that the set thus obtained satisfies the sufficient conditions C0 - C3[6, Chapter 10] (see Appendix A). Picking the seed transition turns out to be an important factor in forming small ample sets, and we have discovered that the transactional nature of many of the systems modeled using the rule-based paradigm allows for a particularly effective choice of the seed transition. These transactional systems often operate in fairly sequential phases of requests, intermediate processing, and grants. Thus, detecting the phase of the transaction in progress allows us to pick, as seed transitions, transitions that will take the current transaction forward, in effect delaying the "scheduling" of new transactions as long as possible. The sequential nature of transactions means that only a very few (often just one) transitions are enabled at any given point in the transaction, resulting in small ample sets.

Roadmap: Section 2 goes over the notations and definitions. In Section 3 , we introduce the notion of exploiting scalarset symmetry, and how it can be used to extrapolate independence results for parameterized systems. We also state and prove our main theorem regarding this result. Section 4 discusses the use of transactions to form more effective ample sets, and Section 5 proposes a novel technique called guard strengthening to soundly refine rule-based systems to achieve higher independence between transitions. Section 6 discusses experiments and results. Related work and conclusions are in Section 7 .

\section{Background, Notations and Definitions}

A labeled finite state transition system $\mathcal{F}$ is a 5 -tuple $\langle S, T, I, P, L\rangle$ where $S$ is a finite set of states, $T$ is a finite set of deterministic transitions, such that every $t \in T$ is a partial function $t: S \mapsto S, I \subseteq S$ is the set of initial states, $P$ is a set of atomic propositions, and $L: S \mapsto 2^{P}$ labels each state with a set of propositions that are true in the state. A labeled path of a finite state system is an infinite sequence starting with a state and then alternating transitions and states,

$$
s_{0}, t_{0}, s_{1}, t_{1}, s_{2}, t_{2}, \ldots,
$$

where $\forall i \geq 0: t_{i}\left(s_{i}\right)=s_{i+1}$. The length of a path is the number of states in the path. A labeled path is called a labeled run if it starts with a state in $I$. For any 
labeled path $p$ of a system, we define the predicate before $\left(p, t_{1}, t_{2}\right)$ to be true when $t_{1}$ occurs before the earliest occurrence of $t_{2}$ in $p$, or $t_{2}$ does not occur in $p$. Let the set of all labelled paths of a finite state system be $\mathcal{P}$. The restriction of $\mathcal{P}$ with respect to a state $s$, written $\mathcal{P}_{\mid s}$, is the set of all labelled paths in $\mathcal{P}$ starting from the state $s$. A transition $t$ is said to be enabled at a state $s$ if $\exists s^{\prime} \in S: t(s)=s^{\prime}$. We define the predicate en $(s, t)$ that is true exactly when $t$ is enabled at $s$. We also define the predicate enabled $(s)=\{t \in T \mid \operatorname{en}(s, t)\}$. Two transitions $t_{1}$ and $t_{2}$ are independent iff the following conditions hold:

- Enabledness(En): $\forall s \in S: \mathbf{e n}\left(s, t_{1}\right) \wedge \mathbf{e n}\left(s, t_{2}\right) \Rightarrow \mathbf{e n}\left(t_{1}(s), t_{2}\right) \wedge \mathbf{e n}\left(t_{2}(s), t_{1}\right)$

- Commutativity $(\mathrm{Co}): \forall s \in S: \mathbf{e n}\left(s, t_{1}\right) \wedge \mathbf{e n}\left(s, t_{2}\right) \Rightarrow t_{1}\left(t_{2}(s)\right)=t_{2}\left(t_{1}(s)\right)$

We define the predicate ind $\left(t_{1}, t_{2}\right)$, that is true exactly when $t_{1}$ and $t_{2}$ are independent, and $\operatorname{dep}\left(t_{1}, t_{2}\right)=\neg \mathbf{i n d}\left(t_{1}, t_{2}\right)$. A property $\pi$ of a system is a formula in next-time free LTL $\left(\operatorname{LTL}_{-X}\right)$, such that the set of propositions in the logic is $P$. For any property $\pi$, we define $\operatorname{props}(\pi) \in 2^{P}$ as the set of propositions occurring in $\pi$. A transition $t$ is invisible with respect to a property $\pi$, written as $\operatorname{inv}_{\pi}(t)$, iff:

$$
\forall s_{1}, s_{2} \in S: t\left(s_{1}\right)=s_{2} \Rightarrow L\left(s_{1}\right) \cap \operatorname{props}(\pi)=L\left(s_{2}\right) \cap \operatorname{props}(\pi)
$$

Our POR algorithm proceeds in two phases, static and dynamic. In the static phase, the dependence matrix is computed, which records the dep relation (and writes it out into a file for use by the dynamic phase of the POR). Computing dep is achieved by symbolically simulating every pair of transitions as described earlier ${ }^{3}$ The POeM (Partial Order enabled Murphi) tool that implements these ideas takes the code fragments defining the guards and actions, and transforms them into equivalent Lisp S-expressions. These are then combined to form Sexpressions representing the enabledness and commutativity relations. We do this over the entire syntax of Murphi, handling loops (by unrolling), and procedures and functions (by in-lining). Each rule of a ruleset is turned not into $N$ separate rules, but just two rules, one called primary and the other called alternate. In a sense these two "fictitious" rules represent all pairwise combinations of the rules that we might otherwise analyze. Then, in our independence analysis, we only perform the following analyses: (i) whether primary is independent of primary, and (ii) whether primary is independent of alternate. This technique is motivated and further explained in Section 3 .

In the dynamic phase of POR, ample sets are constructed as illustrated in Figure 1, during a depth first traversal. We pick an arbitrary enabled transition called seed $4^{4}$ and form an ample set around it, as follows. We first obtain the

${ }^{3}$ In practice, we use conservative approximations when dealing with parameterized transitions, to save on the number of SAT calls, using the notions of primary and alternate.

${ }^{4}$ Later in Section 4, we discuss how these seed transitions are picked according to the weighing scheme described earlier. Also the words 'transition' and 'rule' are synonymous. 


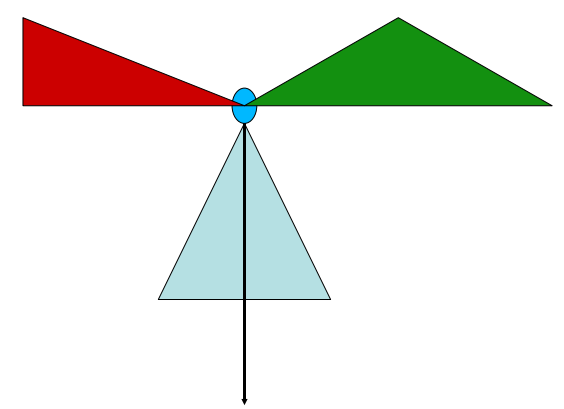

Fig. 1. Ample set computation in POeM

dependency closure of all transitions that are dependent on seed. Now we are left with enabled independent transitions, and disabled dependents (clearly, another possible category, namely disabled independent transitions are completely inconsequential for ample-set formation). We have to ensure (as condition $\mathrm{C} 1$ of $[\underline{6}$, Chapter 10] requires) that there is no transition in the unreduced state graph such that one of these disabled dependent transitions could fire before one of the transitions in the current ample set. This could easily happen if one of the enabled independent transitions could fire and "wake up" one of the disabled dependent transitions. We have experimented with two schemes, the second of which gives better performance:

Approach 1: If the disabled dependent set is empty, then the ample set is the dependency closure set (thus it leaves out the enabled independent transitions), else all enabled transitions are in ample.

Approach 2: If there are any disabled dependent transitions, ensure that they can fire only as a result of any one of the transitions in the dependency closure set firing (thus precluding that they may occur before one of the ample set transitions). This information can be computed and stored in the static phase of the POR algorithm, thus avoiding a run-time cost.

We do have implementations of the other checks, namely $\mathrm{C} 0, \mathrm{C} 2$, and $\mathrm{C} 3$, as 6] requires. In particular, for the $\mathrm{C} 3$ condition which avoids ignoring, we have an implementation that implements an on-the-fly in-stack check. Details of these checks are omitted.

\section{Computing Independence for Parametric Systems}

A Murphi system description is considered to be parameterized if the state variables and transitions are indexed over one or more parameters. In this paper, we only consider parameterized systems with one parameter. In the absence of a general method for verification of parameterized systems, it is common to verify 


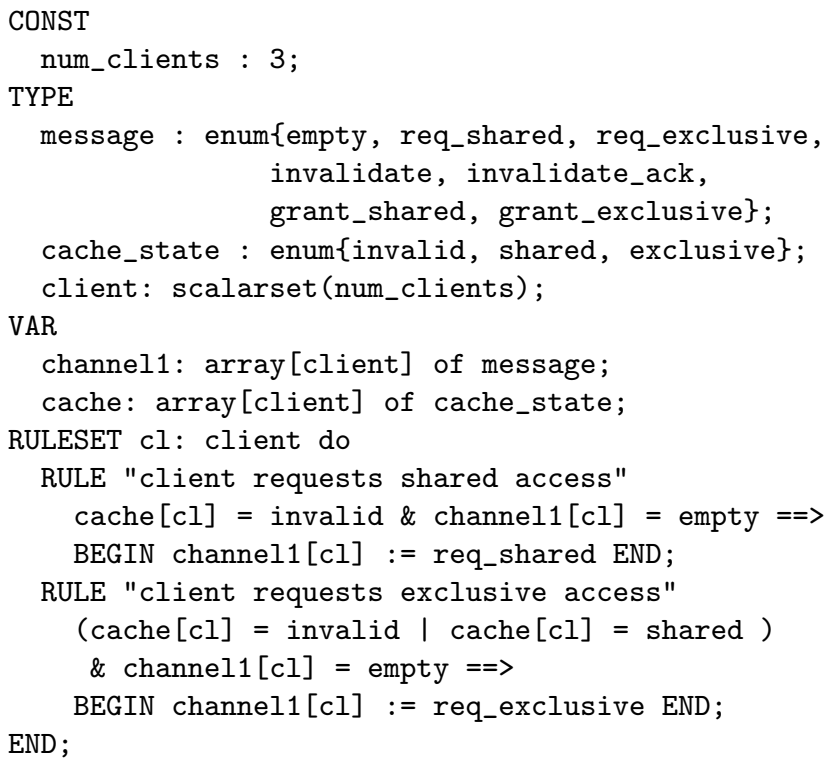

Fig. 2. A simple parameterized Murphi system outline

a system for multiple instances of the parameter. However, realistic system sizes increase greatly with an increase in parameter size, and so does the complexity of computing the independence relation. We show that for such parameterized systems, it is sufficient to compute the independence relation for a small parameter size. Model checking can be performed for higher parameter sizes using this independence relation.

As a simple example, consider the Murphi system outline of Figure 2, This is an extract from the parameterized German protocol, and shows the two transitions responsible for making new requests for access to a cache line, in either the shared or exclusive mode. The parameter of this system is the number of clients, represented by num_clients. The first thing to note is that there are actually multiple instances of each transition (rule) in the system, for any value of num_clients. In this case, there are 3 instances of each rule, corresponding to the range of the variable $\mathrm{cl}$. Theoretically, therefore, we need to check 9 pairs of rules for dependence (each of the 3 instances of the first rule against each of the 3 instances of the second rule). However, as we show in 4 , it suffices to check a pair where the indices have the same value, and a pair where they have different values, and conservatively extrapolate the results to all pairs. So in the given system, we might choose to check rule $1[\mathrm{cl} \leftarrow 1]^{5}$ against rule $2[\mathrm{cl} \leftarrow 1]$ (a pair with the same index value), and rule $1[\mathrm{cl} \leftarrow 1]$ against rule $2[\mathrm{cl} \leftarrow 2]$ (a pair with different index values).

\footnotetext{
${ }^{5}$ the instance of the first rule with $\mathrm{cl}$ set to 1
} 
What does the truth of the above checks for one value of num_clients tell us about the truth of the corresponding checks for a different value of num_clients? Consider first the case of the two rules with the same value for the ruleset parameter $\mathrm{cl}$. Obviously, these two rules are dependent, for they pass neither the enabledness check nor the commutativity check. This is because they are essentially requests from the same node, and therefore each request disables the other. In this case, it would not make any difference what the range of the index variable cl was, since the rules do not count the range in any manner, and only refer to state variables that are directly indexed over $\mathrm{cl}$, which we have already instantiated to a particular value. Therefore, for these particular rules, it is sufficient to check independence for a particular instance, to be able to conclude that for every instance of the parameterized system, instantiations of these rules with the same index value will never be independent. Now consider the case of the two rules with different values for the parameter cl. In this case, the rules involve entirely disjoint sets of state variables, and hence the rules are independent. However, this is true as long as the values of $\mathrm{cl}$ are different for the two rules, irrespective of what particular values they are. Therefore, here too, it is sufficient to check independence for one instance, and infer independence for all instances.

In the following sections, we develop a framework for describing independence among rules of parameterized systems, and show that under certain assumptions, independence of rules is indeed unaffected by parameter size. Section 3.1 introduces the notation and definitions used, and in Section 3.2 we state and prove the main theorem that relates independence of rules across different instances of a parameterized system.

\subsection{Notations and Definitions}

A parameterized Murphi specification can be described in terms of a first order language over the set of variables of the specification. Following Pnueli et al's notion of bounded data systems [14, we partition the set of variables into three broad classes, as follows:

- $\mathcal{V}_{1}=\left\{x_{1}, x_{2}, \ldots x_{a}\right\}$ where $x_{i}$ is interpreted over $\mathbb{B}$, the boolean domain, and $a \in \mathbb{N}$, the set of natural numbers.

$-\mathcal{V}_{2}=\left\{y_{1}, y_{2}, \ldots y_{b}\right\}$ where each $y_{i}$ is a scalarset variable interpreted over the integer subrange $[1 \ldots N]$, and $b \in \mathbb{N}$.

$-\mathcal{V}_{3}=\left\{a r_{1}, a r_{2}, \ldots a r_{c}\right\}$ where each $a r_{i}$ is an array with index type $[1 \ldots N]$, each array's cell type is interpreted over $\mathbb{B}$, and $c \in \mathbb{N}$.

The terms of the language are the boolean constants True and False, variables of type $\mathcal{V}_{1}$ or $\mathcal{V}_{2}$, and array references of the form $\operatorname{ar}_{i}\left[y_{j}\right]$, where $y_{j} \in \mathcal{V}_{2}$. The valid atomic formulas of our language are partitioned into the set of ordinary atomic formulas $\mathcal{O}$ and quantified atomic formulas $\mathcal{Q}$, where: $\mathcal{O}=$ $\left\{x_{i} \mid x_{i} \in \mathcal{V}_{1}\right\} \cup\left\{a r_{i}\left[y_{j}\right] \mid a r_{i} \in \mathcal{V}_{3}, y_{j} \in \mathcal{V}_{2}\right\} \cup\left\{y_{i}=y_{j} \mid y_{i}, y_{j} \in \mathcal{V}_{2}\right\}$, and $\mathcal{Q}=\left\{\forall x . a r_{i}[x] \mid a r_{i} \in \mathcal{V}_{3}, 1 \leq x \leq N\right\} \cup\left\{\forall x . \neg a r_{i}[x] \mid a r_{i} \in \mathcal{V}_{3}, 1 \leq x \leq N\right\}$ 
$\cup\left\{\exists x . a r_{i}[x] \mid a r_{i} \in \mathcal{V}_{3}, 1 \leq x \leq N\right\} \cup\left\{\exists x . \neg a r_{i}[x] \mid a r_{i} \in \mathcal{V}_{3}, 1 \leq x \leq N\right\}$. The set of formulas is then the standard extension of the atomic formulas using the boolean connectives $\wedge, \vee$ and $\neg$. We say that the set of all formulas over a set of variables $\mathcal{V}, \mathcal{L}(\mathcal{V})$ is the language of our logic.

A Murphi system description, which consists of a set of variable declarations, and a set of transitions (rules) defined as guard/action pairs, can be mapped into our first order language by mapping the variable definitions to the variables of the language, mapping guards to formulas of the language, and mapping actions as sets of substitutions of variables by terms or formulas ${ }^{6}$ of the language. For a complete description of the allowed substitutions, and the corresponding Murphi constructs, see [3. A state of a Murphi system can thus be seen as an interpretation of the variables of the logic. We denote the set of all states of a system as $S$, and, in particular, the set of all states of a parameterized system with parameter $N$ as $S(N)$.

In bounded data systems, both states (interpretations) and the satisfaction of formulas, are symmetric with respect to any permutation of the indices [14]. This is enforced in Murphi syntax by declaring the parameter range to be a scalarset type.

\subsection{The Carry Over Theorem}

We now show that in the above setting, we can compute the dependence relation between transitions for all parameter sizes $N>1$, by computing the relation for a small size, calculated as described below.

Enabledness: Given a pair of rules $\left\langle g_{1}, a_{1}\right\rangle$ and $\left\langle g_{2}, a_{2}\right\rangle$, they satisfy the enabledness condition when:

$$
g_{1}(s) \wedge g_{2}(s) \Rightarrow g_{1}\left(a_{2}(s)\right) \wedge g_{2}\left(a_{1}(s)\right)
$$

is valid over $S(N)$, the set of all states (interpretations) $s, g_{1}(s)$ denotes the evaluation of the formula $g_{1}$, given the interpretation $s$ of the variables, $a_{1}(s)$ (with a slight abuse of notation) denotes the application of the substitutions represented by $a_{1}$ to the variables, followed by an evaluation of the resulting terms over the interpretation $s$. Similarly for $g_{2}$ and $a_{2}$.

We would like to find a bound, $\widehat{N}$, such that 3.1 is valid over $S(N)$ for all $N$, $N>1$ iff it is valid over $S(N)$ for all $N, 1<N \leq N$. To arrive at such a bound, we proceed as follows: in formula 3.1, we push negations inside atomic formulas of type $\mathcal{Q}$ (ie, a formula $\neg \forall i . a r_{j}[i]$ is converted into the equivalent formula $\exists i . \neg a r_{j}[i]$, and so on for every atomic formula of type $\left.\mathcal{Q}\right)$. Let the cardinality of the set $\mathcal{V}_{2}$ be $k$, the number of existentially quantified atomic formulas of type $\mathcal{Q}$ in a guard $g_{i}$ be $e_{i}$, and the number of universally quantified atomic formulas of type $\mathcal{Q}$ in $g_{i}$ be $u_{i}$.

\footnotetext{
${ }^{6}$ Since the variables in $\mathcal{V}_{1}$ and $\mathcal{V}_{3}$ are of boolean type, they can be assigned any valid formula of the language, because Murphi allows arbitrary boolean expressions as rvalues in assignments.
} 
Theorem 1. 3.1 is valid over $S(N)$ for all $N, N>1$ iff it is valid over $S(N)$ for all $N, 1<N \leq \widehat{N}$, where $\widehat{N}=b+2\left(\max _{1<i<2} e_{i}+\max _{1<i<2} u_{i}\right)$.

Proof (sketch) 7 To show this, it is sufficient to show that the negation of 3.1

$$
g_{1}(s) \wedge g_{2}(s) \wedge\left(\neg g_{1}\left(a_{2}(s)\right) \vee \neg g_{2}\left(a_{1}(s)\right)\right)
$$

is satisfiable for $N>\widehat{N}$ iff it is satisfiable for some $N, 1<N \leq \widehat{N}$. To show this, moreover, it is sufficient to show that if 3.2 is satisfiable over $S(N)$, for $N>\widehat{N}$, it is satisfiable over $S(\widehat{N})$.

By counting the number of existentially quantified atomic formulas of the forms $\exists x . a r_{i}[x]$ or $\exists x . n e g a r_{i}[x]$ in 3.2 , which can be equivalently written as $a r_{i}[p]$ and $\neg a r_{i}[q]$ respectively, where $p$ and $q$ are fresh variables of type $\mathcal{V}_{2}$, we can show that the total number of variables of type $\mathcal{V}_{2}$ is bounded by $\widehat{N}$. Thus, given an interpretation $s$ over $S(N)$ that satisfies 3.2 it can assign at most $\alpha \leq \widehat{N}$ different values to these variables. Without loss of generality, assume that these values are $v_{1}<v_{2}<\cdots<v_{\alpha}$. Since the system is symmetric, there is a permutation over the indices $[1 . . N]$ that maps $v_{k}$ to $k$, for every $k \in 1 . . \alpha$. Let $\tilde{s}$ be the state derived from $s$ by applying this permutation-induced transformation to the set of variables above. Clearly, $\tilde{s}$ is also an interpretation that satisfies 3.2 . To construct the interpretation $\hat{s} \in \widehat{N}$ that satisfies 3.2 , we let $\tilde{s}$ and $\hat{s}$ agree on the interpretation of the variables in $V_{1}$ and $V_{2}$. For the remaining variables $a r_{1}, a r_{2}, \ldots a r_{c}$, we let $\tilde{s}$ and $\hat{s}$ agree on the values of all $a r_{i}[k]$, for $k \leq \alpha$. After replacing existentials by new variables, the formula 3.2 is a formula over the variables in $V_{1}$ and $V_{2}$, and universally (over the parameter size) quantified expressions over $V_{3}$. Since $\tilde{s}$ and $\hat{s}$ agree on the interpretation of all of the above, $\hat{s}$ satisfies 3.2 over $S(\widehat{N})$. By similar reasoning, we can also compute bounds on the commutativity condition. Taken together, these provide an overall bound on the size of the system for which independence checks need to be performed for partial order reduction.

\section{Transaction-based priorities for Ample Set Construction}

For many of the kinds of systems that are typically described using the rulebased paradigm (e.g., protocols of various types), it is often the case that the system proceeds along fairly sequential paths called transactions. For example, consider a typical directory-based cache coherence protocol. Most activities in such protocols begin with the cache controller making a request for a line. This request travels to the directory controller which typically evicts other caches from the sharing group by sending invalidations. Thereafter, the directory controller sends the line back to the requesting node. Modeled in Murphi, we can

\footnotetext{
7 The proof is very similar to, and follows closely, the proof of Claim 3 in [14, Section $4]$
} 
say that (i) this whole activity consists of a transaction (refer to [13] for somewhat related notions of a transaction), (ii) there are Murphi rules that begin a transaction, there are rules that are somewhere in the "middle" of transactions (e.g., invalidation rules), and finally there are rules that end transactions. One can often obtain the situation of a rule - whether it is at the beginning, middle, or end of a transaction - through concrete execution on small instances of the protocol. Most designers also clearly know the situation of rules. In any case, we weigh each rule as follows: (i) rules that begin transactions are weighed "low," (ii) the rules that end transactions are weighed "high," (iii) rules that are in the middle of transactions are weighed "medium." We need not be exact in how we assign numeric values to "low, medium, and high." Users can be completely wrong in these weight assignments - the only consequence being poorer ample sets but never incorrect execution.

\section{$5 \quad$ Strengthening guards}

It is often the case that a pair of rules is independent at all reachable states, but dependent at some unreachable state(s). Our current analysis marks such rules dependent, since it starts from an entirely general symbolic state. To be able to use the independence of these rules during partial order reduction, a simple idea is to find potential strengthenings for guards, that don't change the enabledness of rules in reachable states, and extend the independence of rules to all states, both reachable and unreachable. This is useful while checking the $\mathbf{C 1}$ condition, since the fewer the dependent transitions, the smaller the likelihood of there being disabled transitions dependent on the ample set.

To actually discover these strengthenings requires a deep understanding of the protocol involved, and we discuss some intuitions in Section 6 .

Once we have strengthened the guards of transitions, it is necessary to show that these strengthenings are sound, and do indeed preserve the semantics of the original transitions. We now show that it is sufficient to model check the strengthened system with a modified property, to be able to prove the soundness of the strengthenings.

Since Murphi transitions are guard action pairs $\left(g_{i}, a_{i}\right)$, strengthening the guards corresponds to adding predicates $p_{i}$ to the guards of transitions $t_{i}$. Define the strengthening operator $\Theta$ over transitions such that:

$$
\Theta\left(\left\langle g_{i}, a_{i}\right\rangle\right)= \begin{cases}\left\langle g_{i} \wedge p_{i}, a_{i}\right\rangle & \text { if } t_{i} \text { is strengthened } \\ \left\langle g_{i}, a_{i}\right\rangle & \text { otherwise }\end{cases}
$$

We extend $\Theta$ to apply to runs $\sigma=\left\langle s_{1}, t_{1}, s_{2}, \ldots, s_{k}\right\rangle$ so that $\Theta(\sigma)$ results in the sequence (not necessarily a run) $\left\langle s_{1}, \Theta\left(t_{1}\right), s_{2}, \ldots, s_{k}\right\rangle$. Let the original system be $\mathcal{F}$, and the modified system $\mathcal{F}^{\prime}$. Note that both systems have the same set of states, and the same initial state predicate $I$. Assume that the property to be verified of the original system was $P$. We model check the new system with the property $P \wedge S t r$, where:

$$
S t r=\left(g_{i_{1}} \rightarrow p_{i_{1}}\right) \wedge\left(g_{i_{2}} \rightarrow p_{i_{2}}\right) \wedge \ldots \wedge\left(g_{i_{k}} \rightarrow p_{i_{k}}\right)
$$


$g_{i_{1}} \ldots g_{i_{k}}$ are the $k$ guards of the original system that have been strengthened with the predicates $p_{i_{1}} \ldots p_{i_{k}}$.

\section{Theorem 2.}

$$
\mathcal{F}^{\prime} \models P \wedge S t r \Rightarrow \mathcal{F} \models P
$$

Proof: By contradiction. Assume that the antecedent of the theorem is true, and assume that there is a run $r=\left\langle s_{1}, t_{1}, s_{2}, t_{2}, \ldots, s_{m}\right\rangle$ of $\mathcal{F}$ that does not satisfy the property $P$. Without loss of generality, we assume that $s_{1}, s_{2}, \ldots s_{m-1} \models P$, and $s_{m} \not \models P$. If $\Theta(r)$ is a run of $\mathcal{F}^{\prime}, s_{m} \models P \wedge S t r$, which implies that $s_{m}=P$, contradicting our assumption. Therefore, assume that $\Theta(r)$ is not a run of $\mathcal{F}^{\prime}$. Then, there is a $t_{k}$, such that $\Theta\left(t_{k}\right)$ is not enabled at $s_{k}$, and $\Theta\left(\left\langle s_{1}, t_{1}, s_{2}, \ldots s_{k}\right\rangle\right)$ is a run of $\mathcal{F}^{\prime}$. Since $r$ is a run of $\mathcal{F}, t_{k}$ is enabled at $s_{k}$.

Case 1: $\Theta\left(t_{k}\right)=\left\langle g_{k}, a_{k}\right\rangle$. In this case, since $\Theta\left(t_{k}\right)$ is not enabled at $s_{k}$, this implies that $s_{p} \not \models g_{k}$. But we know that $t_{k}$ is enabled at $s_{k}$. That is, $s_{k} \models g_{k}$, leading to a contradiction.

Case 2: $\Theta\left(t_{k}\right)=\left\langle g_{k} \wedge p_{k}, a_{k}\right\rangle$. In this case, we have $s_{k} \not \models g_{k} \wedge p_{k}$. However, we know that $s_{k}=g_{k}$. Also, since $\Theta\left(\left\langle s_{1}, t_{1}, s_{2}, \ldots s_{k}\right\rangle\right)$ is a run of $\mathcal{F}^{\prime}, s_{k} \models P \wedge S t r$. That is, $s_{k}=g_{k} \rightarrow p_{k}$. Therefore, $s_{k} \models g_{k} \wedge p_{k}$, leading to a contradiction.

Thus, in every case, we arrive at a contradiction, and hence, the theorem is true, and, by model checking the strengthened system for the property $P \wedge S t r$, we can prove that the strengthenings of the guards are sound.

If the model check fails, on the other hand, we have to manually examine the error trail to determine which of the strengthenings does not hold, and rerun the model check after making the necessary changes.

\section{Experimental Results, and Analysis}

We have run POeM on a number of examples of different sizes, and Table 1 shows our overall results on some mutual exclusion algorithms and a cache coherence protocol. The experiments in Table 1 were performed with Murphi's symmetry reduction turned on, whenever scalar sets were employed. This is because symmetry and partial order reduction are a safe combination for safety property verification 8 . Guard strengthening and transaction-based weights were not employed in these examples, and are discussed later. In the table, the columns under "Unreduced" represent the number of states explored, and the time taken for the verification to complete, without any partial order reduction. The columns under "Static PO" represent the same figures for the case where a static, syntaxbased analysis was used to determine the independence relation (this was our initial prototype version of POeM before we moved on to the use of SAT for independence computation). The columns under "Symbolic PO" represent the figures for POeM. The final column, "Analysis Time", is the time taken by POeM's symbolic evaluation based module to compute the independence relation. As can be seen, POeM is most effective on large examples, where the overhead of performing the symbolic analysis, and computing an ample set at each state, is outweighed by the savings that result from a far fewer number of states being explored. 


\begin{tabular}{|c|c|c|c|c|c|c|c|}
\hline \multirow[t]{2}{*}{ Example } & \multicolumn{2}{|c|}{ Unreduced } & \multicolumn{2}{|c|}{ Static PO } & \multicolumn{2}{|c|}{ Symbolic PO } & \multirow{2}{*}{$\begin{array}{c}\text { Analysis } \\
\text { Time }\end{array}$} \\
\hline & States & Time & States & Time & States & Time & \\
\hline Bakery & 157 & 0.1 & 157 & 0.1 & 119 & 0.1 & 8.9 \\
\hline Burns & 82010 & 1.83 & 82010 & 3.65 & 69815 & 11.67 & 52.9 \\
\hline Dekker & 100 & 0.13 & 100 & 0.13 & 90 & 0.13 & 11.6 \\
\hline Dijkstra6 & 11664 & 0.57 & 11664 & 0.88 & 4900 & 1.17 & 17.9 \\
\hline Dijkstra8 & 139968 & 4.32 & 139968 & 8.81 & 33286 & 8.98 & $0^{8}$ \\
\hline Dijkstra1d & $>1.5 \mathrm{M}$ & $>1000.0$ & $>1.5 \mathrm{M}$ & $>1000.0$ & 202248 & 82.6 & 0 \\
\hline \begin{tabular}{|l|}
$\mathrm{DP} 6$ \\
\end{tabular} & 1152 & 0.32 & 1152 & 0.36 & 90 & 0.31 & 0 \\
\hline DP10 & 125952 & 7.44 & 125952 & 7.86 & 823 & 0.48 & 0 \\
\hline DP14 & $>1.0 \mathrm{M}$ & $>800.0$ & $>1.0 \mathrm{M}$ & $>800.0$ & 7395 & 2.6 & 0 \\
\hline Peterson2 & 26 & 0.15 & 26 & 0.15 & 24 & 0.15 & 4.6 \\
\hline Peterson4 & 22281 & 0.3 & 22281 & 0.53 & 14721 & 0.58 & 0 \\
\hline German6 & 7378 & 1.31 & 7378 & 1.36 & 2542 & 0.83 & 32.4 \\
\hline German8 & 42717 & 14.6 & 42717 & 15.23 & 10827 & 4.6 & 0 \\
\hline German10 & 193790 & 127.24 & 193790 & 131.83 & 36606 & 24.91 & 0 \\
\hline Leader1 & 683 & 0.32 & 683 & 0.36 & 21 & 0.10 & 8.5 \\
\hline Leader2 & 12651 & 0.20 & 12651 & 0.33 & 12651 & 0.33 & 4.6 \\
\hline
\end{tabular}

Table 1. Performance of partial order reduction algorithm

Assessing Guard Strengthenings: We experimented with guard strengthenings on the German protocol, as well as the FLASH cache coherence protocol, and these results are now discussed. The German cache coherence protocol is a directorybased protocol for maintaining coherence among shared memory multiprocessors, proposed by Steven German [9] The Murphi description of the protocol only models a single address/cache line, and a parameterized number of processors.

Our technique for generating predicates to strengthen guards is to first run POeM directly on the protocol, and analyze the resulting dependency matrix. For pairs of rules that POeM marks dependent, we examine the test(s) that failed (enabledness, dependency, or both), and try to reason about predicates that, if added, would make the rules independent, without violating the properties we wish the protocol to hold. If we are able to come up with such predicates, we add them to the guard, and add the corresponding implication predicate to the invariant to be proved.

Run directly on the German protocol, POeM concludes that the rule "home sends invalidate message" is dependent on the rule "home sends reply to client - exclusive".

The guards for the two rules are:

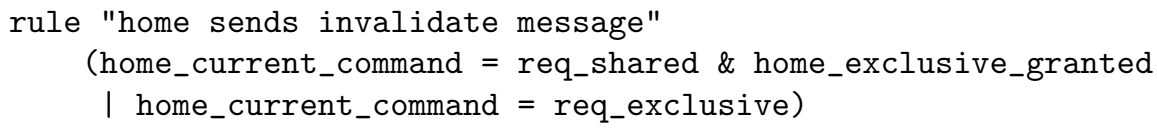

${ }^{8} 0$ indicates that the Carry Over Theorem of Section 3.2 was used. 


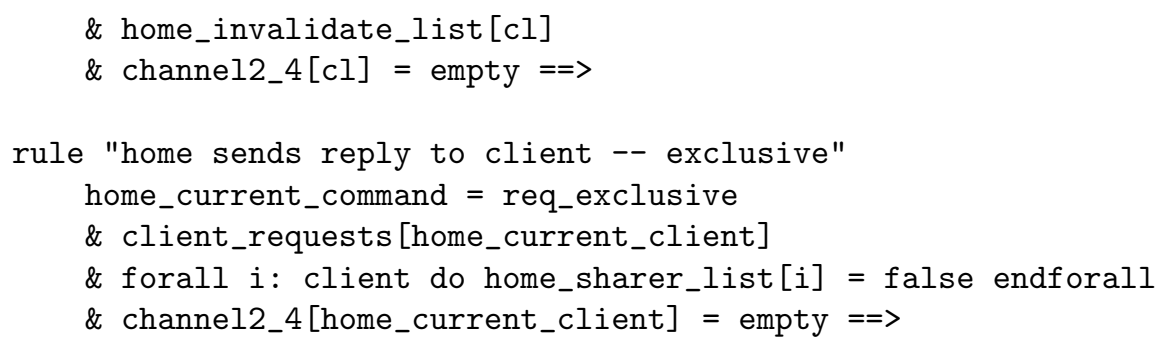

It is evident that the two rules ought never to be enabled together, and therefore, marked independent. However, it is not apparent what predicate is to be added to enforce this. It is clear from the existing guards that, if the rules are to be simultaneously enabled, home_current_command = req_exclusive must be true. Looking at the rule "home picks new request", which sets this variable, leads to the realization that, in the case of a request for exclusive access, the home node copies the home_sharer_list to the home_invalidate_list. The protocol then clears an entry in the invalidate list once it sends out the invalidate message to that client, and clears the entry in the sharer list once the client has sent the acknowledgment to the invalidate. This means that, at the time the home node sends out an invalidate message to a client, that client must be on the sharer and invalidate lists. Therefore, we can add the predicate home_sharer_list [cl] to the guard for the rule "home sends invalidate message":

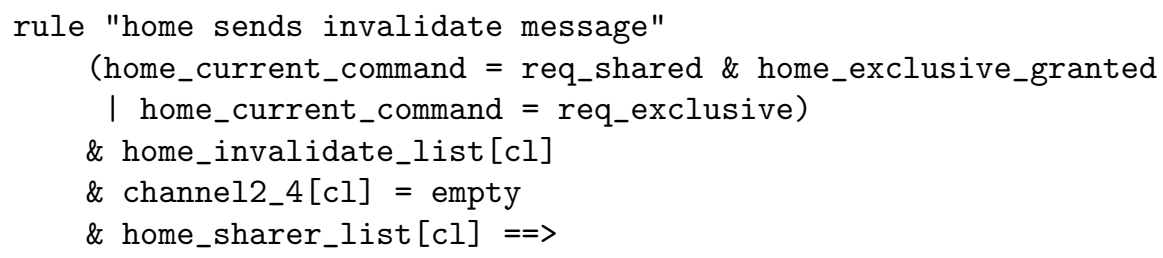

Similar reasoning is used to strengthen the guards of other pairs of rules that we determine to have been falsely marked dependent by POeM, resulting in the final strengthened version in the appendix.

As is evident, the ability to effectively strengthen the guards of a given protocol depends on a good understanding of the workings of the protocol, which we possess for the German protocol. From a practical perspective, however, industrial design groups possess a deep understanding of their protocols, and we have reasons to believe that designers will, when presented with false entries in the independence matrix, be able to identify guard strengthenings as discussed above. Our results on the FLASH coherence protocol further demonstrate the effectiveness of this approach, yielding over $60 \%$ reduction for 4 nodes, although, with over 30 rules, and many auxiliary variables, it is a much more complex protocol and guard strengthenings were only performed for the most obvious cases.

Table 2 shows the results of running the protocols with and without strengthened guards. 


\begin{tabular}{|l|r|r|r|r|r|r|r|r|}
\hline Example & \multicolumn{3}{|c|}{ Without Strengthening } & \multicolumn{3}{c|}{ With Strengthening } \\
& \multicolumn{2}{|c|}{ Unreduced } & \multicolumn{2}{|c|}{ POeM } & \multicolumn{2}{c|}{ Unreduced } & \multicolumn{2}{c|}{ POeM } \\
& States & Time & States & Time & States & Time & States & Time \\
\hline German6 & 13270 & 2.68 & 4485 & 1.29 & 7378 & 1.42 & 2542 & 0.74 \\
\hline German8 & 81413 & 30.28 & 20104 & 8.87 & 42717 & 14.5 & 10827 & 4.56 \\
\hline German10 & 378236 & 260.96 & 69613 & 49.04 & 193790 & 126.6 & 36606 & 24.72 \\
\hline FLASH & 6336 & 0.78 & 6336 & 1.46 & 2888 & 0.46 & 2146 & 0.64 \\
\hline
\end{tabular}

Table 2. Advantages of Guard Strengthening

Assessing Transaction-based Priorities: The next set of experiments run on the German protocol were aimed at testing the significance of user-defined priorities for rules, over the automatically computed priorities, which are based on the number of variable references. Table 3 shows the comparison between the two methods of assigning priorities to rules. Lower weights translate into a higher priority for the rule to be picked as the seed transition.

\begin{tabular}{|c|c|c|c|c|c|c|c|c|}
\hline \multirow[t]{3}{*}{ Ex } & \multicolumn{4}{|c|}{ Without Strengthening } & \multicolumn{4}{|c|}{ With Strengthening } \\
\hline & \multicolumn{2}{|c|}{ User defined wts } & \multirow{2}{*}{\multicolumn{2}{|c|}{$\begin{array}{l}\text { Varcount based wts } \\
\text { States } \quad \text { Time }\end{array}$}} & \multicolumn{2}{|c|}{ User defined wts } & \multicolumn{2}{|c|}{ Varcount based wts } \\
\hline & States & Time & & & States & Time & States & Time \\
\hline G6 & 2521 & 0.66 & 4485 & 1.29 & 1166 & 0.36 & 2542 & 0.74 \\
\hline G8 & 8098 & 2.75 & 20104 & 8.87 & 2851 & 0.94 & 10827 & 4.56 \\
\hline G10 & 20968 & 10.52 & 69613 & 49.04 & 5890 & 2.57 & 36606 & 24.72 \\
\hline
\end{tabular}

Table 3. Transaction-based priorities vs. Variable reference based priorities. ( $\mathrm{G}=$ German.)

The user defined priorities were assigned in such a fashion as to give higher priority to rules that complete transactions, the transactions in this case being the requests for exclusive or shared access to a line. Rules that represent the intermediate steps of a transaction were given medium priority, and rules that represent the start of a transaction were given the lowest priority.

In the case of the German protocol, user-defined priorities gave a distinct performance boost to POeM, resulting in upto an $80 \%$ reduction over the already reduced state space explored by POeM using the regular variable reference count based priorities. This confirms our intuitions that user-defined priorities are a good way to select the seed transition around which to form ample sets.

Recently [5], we have built an experimental variant of Murphi that records the sequence of rules fired with respect to user-identified request rules and completion rules. From this experimental version of Murphi, we observe that rule weights can be computed with reasonable accuracy based on concrete executions of small instances of the protocol. 


\subsection{Protocols that yield low reductions with POeM}

Our partial order reduction algorithm yields large reductions on many complex protocols, but also fails to yield significant reductions on some others. An example in Table 1 is the leader election protocol from [6](Leader2). This example is of a network of nodes in a ring topology running an algorithm to determine the node with the largest id. The algorithm involves exchanging messages through buffers, and POeM's independence computation, relying on the primary and alternate checks, concludes that all the message-passing transitions of each node are dependent on those of all of the other nodes, although each node's transitions only depend on its neighbor's transitions (since neighboring nodes read and write a common message buffer). This indicates that rule-based systems might benefit from making buffers/queues first-class data structures in their language, allowing partial order reduction algorithms to take advantage of the orchestrated fashion in which these buffers operate. On the other hand, POeM is very successful on the other leader election example studied (Leader1), since that example employs a single-cell buffer, and thus forces the nodes to proceed in lock-step fashion. This also makes the case that the specification style can often influence the amount of reduction achievable.

\section{Conclusions and Future Directions}

In this paper, we have described in detail a number of heuristics and techniques to further improve the efficiency of partial order reduction algorithms for rule-based systems, and demonstrated the advantages of our heuristics over conventional, static analysis based partial order reduction algorithms for these types of systems.

An interesting experiment to perform might be to replace our SAT-based backend with a more powerful solver such as CVC [15], which combines decision procedures for fragments of arithmetic and so on. It might also be possible to automatically generate candidate predicates to strengthen guards, based on the satisfying assignment returned by the SAT solver/decision procedure, in case two rules are found to be dependent.

\section{APPENDIX}

\section{A Sufficient conditions for Ample Set Construction}

Adapted from [6, Chapter 10], the sufficient conditions C0-C3 for constructing valid ample sets are:

- C0 : $\forall s \in S: \operatorname{ample}(s)=\phi \Leftrightarrow \operatorname{enabled}(s)=\phi$. An ample set is empty if and only if there are no enabled transitions.

- C1: $\forall s \in S: \forall t_{1}, t_{2} \in T: t_{1} \in \operatorname{ample}(s) \wedge t_{2} \notin \operatorname{ample}(s) \wedge \operatorname{dep}\left(t_{1}, t_{2}\right) \Rightarrow$ $\forall p \in \mathcal{P}_{\mid s}: \exists t_{3} \in \operatorname{ample}(s): \operatorname{before}\left(p, t_{3}, t_{2}\right)$ Along every path in the full 
state graph that starts at state $s$, the following must hold - if there is an enabled transition that depends on a transition in the ample set, it is not taken before some transition from the ample set is taken.

- C2 : $\forall s \in S: \operatorname{ample}(s) \neq \operatorname{enabled}(s) \Rightarrow \forall t \in \operatorname{ample}(s): \operatorname{inv}_{\pi}(t)$. If a state is not fully expanded, then every transition in the ample set is invisible.

- C3 $3^{9}: \forall s \in S: \operatorname{ample}(s) \neq \operatorname{enabled}(s) \Rightarrow \exists t \in \operatorname{ample}(s): t(s) \notin$ $\operatorname{onstack}(s)$ There is no transition t that is enabled in a state that is part of a cycle, and is not in the ample set of any state in that cycle.

\section{References}

1. Arvind. Bluespec: A language for hardware design, simulation, synthesis and verification invited talk. In MEMOCODE, pages 249-, 2003.

2. R. Bhattacharya, S. German, and G. Gopalakrishnan. Symbolic partial order reduction for rule based transition systems. In CHARME '05, October 2005.

3. Ritwik Bhattacharya. http://www.cs.utah.edu/ ritwik/carryover.html.

4. Ritwik Bhattacharya, Steven German, and Ganesh Gopalakrishnan. A symbolic partial order reduction algorithm for rule based transition systems. Technical Report UUCS-03-028, School of Computing, University of Utah, 2003.

5. Xiaofang Chen. personal communication.

6. Edmund M. Clarke, Orna Grumberg, and Doron Peled. Model Checking. MIT Press, December 1999.

7. David Dill. The stanford murphi verifier. In Computer Aided Verification, pages 390-393, 1996.

8. E. Allen Emerson, Somesh Jha, and Doron Peled. Combining partial order and symmetry reductions. In TACAS '97, pages 19-34, 1997.

9. Steven German. personal communication.

10. G.J. Holzmann, P. Godefroid, and D. Pirottin. Coverage preserving reduction strategies for reachability analysis. In Proc. 12th Int. Conf on Protocol Specification, Testing, and Verification, INWG/IFIP, Orlando, Fl., June 1992.

11. C. Norris Ip and David L. Dill. Better verification through symmetry. In Int'l Conference on Computer Hardware Description Language, 1993.

12. L. Lamport. Specifying concurrent systems with tla+, 1999.

13. Vladimir Levin, Robert Palmer, Shaz Qadeer, and Sriram K. Rajamani. Sound transaction-based reduction without cycle detection. In SPIN, pages 106-122, 2005.

14. Amir Pnueli, Sitvanit Ruah, and Lenore D. Zuck. Automatic deductive verification with invisible invariants. In TACAS, pages 82-97, 2001.

15. Aaron Stump, Clark W. Barrett, and David L. Dill. CVC: A Cooperating Validity Checker. In $C A V$, pages 500-504, 2002.

\footnotetext{
${ }^{9}$ For a proof of the sufficiency of this form of the condition see 10
} 